\title{
MXene/铜合金复合材料的制备与性能研究
}

\author{
司晓阳，陈凡燕，邓启煌，都时禹，黄 庆 \\ (中国科学院 宁波材料技术与工程研究所 特种纤维与核能材料工程实验室, 宁波 315201)
}

摘 要: 采用分子级混合方法及 SPS 烧结技术制备了 $\mathrm{Ti}_{3} \mathrm{C}_{2} \mathrm{~T}_{x}$ 含量分别为 $5 \mathrm{vol} \% 、 10 \mathrm{vol} \%$ 和 $20 \mathrm{vol} \%$ 的 $\mathrm{Cu} / \mathrm{Ti}_{3} \mathrm{C}_{2} \mathrm{~T}_{x}$ 复 合材料, 研究了 $\mathrm{Ti}_{3} \mathrm{C}_{2} \mathrm{~T}_{x}$ 含量对铜基复合材料的导电性、力学性能及摩擦磨损性能的影响。研究发现: 随 $\mathrm{Ti}_{3} \mathrm{C}_{2} \mathrm{~T}_{x}$ 含量增加, $\mathrm{Cu} / \mathrm{Ti}_{3} \mathrm{C}_{2} \mathrm{~T}_{x}$ 复合材料的相对密度及电导率均持续下降, 拉伸强度则先升高后下降; 当 $\mathrm{Ti}_{3} \mathrm{C}_{2} \mathrm{~T}_{x}$ 含量为 $5 \mathrm{vol} \%$ 时, $\mathrm{Cu} / \mathrm{Ti}_{3} \mathrm{C}_{2} \mathrm{~T}_{x}$ 复合材料的拉伸强度相比纯铜提高了 $43 \%$ 。添加 $\mathrm{Ti}_{3} \mathrm{C}_{2} \mathrm{~T}_{x}$ 可以明显改善 $\mathrm{Cu} / \mathrm{Ti}_{3} \mathrm{C}_{2} \mathrm{~T}_{x}$ 复合材料的 摩擦磨损性能, 当 $\mathrm{Ti}_{3} \mathrm{C}_{2} \mathrm{~T}_{x}$ 含量为 $10 \mathrm{vol} \%$ 时, $\mathrm{Cu} / \mathrm{Ti}_{3} \mathrm{C}_{2} \mathrm{~T}_{x}$ 复合材料的磨损率仅为 $2.55 \times 10^{-7} \mathrm{~mm}^{3} /(\mathrm{N} \cdot \mathrm{m})$, 比纯铜降低 了一个数量级。

关 键 词: $\mathrm{Ti}_{3} \mathrm{C}_{2} \mathrm{~T}_{x}$; 铜基复合材料; 导电; 摩擦磨损

中图分类号: TB333 文献标识码: A

\section{Preparation and Property of MXene/Copper Alloy Composites}

\author{
SI Xiao-Yang, CHEN Fan-Yan, DENG Qi-Huang, DU Shi-Yu, HUANG Qing
}

(Engineering Laboratory of Specialty Fibers and Nuclear Energy Materials (FiNE), Ningbo Institute of Industrial Technology, Chinese Academy of Sciences, Ningbo 315201, China)

\begin{abstract}
Cu} / \mathrm{Ti}_{3} \mathrm{C}_{2} \mathrm{~T}_{x}$ composites with $\mathrm{Ti}_{3} \mathrm{C}_{2} \mathrm{~T}_{x}$ content of $5 \mathrm{vol} \%, 10 \mathrm{vol} \%$ and $20 \mathrm{vol} \%$ were prepared by using a molecular-level mixing process and spark plasma sintering (SPS). Influence of $\mathrm{Ti}_{3} \mathrm{C}_{2} \mathrm{~T}_{x}$ content on electrical, mechanical and tribological properties was investigated. The result showed that relative density and electrical conductivity of $\mathrm{Cu} / \mathrm{Ti}_{3} \mathrm{C}_{2} \mathrm{~T}_{x}$ composites gradually decreased with the $\mathrm{Ti}_{3} \mathrm{C}_{2} \mathrm{~T}_{x}$ content increase, while the tensile strength of $\mathrm{Cu} / \mathrm{Ti}_{3} \mathrm{C}_{2} \mathrm{~T}_{x}$ composites increased at first and then decreased. When the $\mathrm{Ti}_{3} \mathrm{C}_{2} \mathrm{~T}_{x}$ content was $5 \mathrm{vol} \%$, the tensile strength of $\mathrm{Cu} /$ $\mathrm{Ti}_{3} \mathrm{C}_{2} \mathrm{~T}_{x}$ composites increased by $43 \%$ than that of pure copper. Tribological properties of $\mathrm{Cu} / \mathrm{Ti}_{3} \mathrm{C}_{2} \mathrm{~T}_{x}$ composites were enhanced by the addition of $\mathrm{Ti}_{3} \mathrm{C}_{2} \mathrm{~T}_{x}$. When the $\mathrm{Ti}_{3} \mathrm{C}_{2} \mathrm{~T}_{x}$ content was $10 \mathrm{vol} \%$, the wear rate of $\mathrm{Cu} / \mathrm{Ti}_{3} \mathrm{C}_{2} \mathrm{~T}_{x}$ composites was $2.55 \times 10^{-7} \mathrm{~mm}^{3} /(\mathrm{N} \cdot \mathrm{m})$, which was one magnitude lower than that of pure copper.
\end{abstract}

Key words: $\mathrm{Ti}_{3} \mathrm{C}_{2} \mathrm{~T}_{x}$; copper matrix composites; electrical conductivity; friction and wear

铜及铜合金具良好的塑性和韧性，优异的导电 导热性，易于加工成型和强耐腐蚀性，被广泛应用 于电子电气、机械交通、能源、航空航天及国防工 业等领域。但是它的强度低、耐磨性能差等缺点限 制了其发展。层状材料(如石墨烯、 $\mathrm{MoS}_{2} 、 \mathrm{BN}$ 等) 因具有特殊的结构和性能, 被大量应用于改善铜基 复合材料的性能 ${ }^{[1-4]}$ 。Chen 等 ${ }^{[5]}$ 通过分子级混合技术
制备了石墨烯分散均匀的铜/石墨烯复合材料，通过 添加石墨烯可以明显改善复合材料的力学及摩擦磨 损性能。

MXenes 材料是一种近年来发展起来的二维层 状结构的前过渡金属碳化物和碳氮化合物，对应的 三元体相材料为 MAX 相 ${ }^{[6]}$ 。MAX 相是一种三元层 状结构碳化物或氮化物材料, 其结构通式为 $\mathrm{M}_{n+1} \mathrm{AX}_{n}$

收稿日期: 2017-06-12; 收到修改稿日期：2017-12-10

基金项目：宁波市石墨烯重大专项(2014S10001) Ningbo Municipal Key Project (2014S10001)

作者简介: 司晓阳(1991-), 女, 硕士研究生. E-mail: sixiaoyang@nimte.ac.cn

通讯作者: 黄 庆, 研究员. E-mail: huangqing@nimte.ac.cn 
$(n=1,2$ 或 3), 其中 $\mathrm{M}$ 代表前过渡金属元素, $\mathrm{A}$ 代表 主族元素(主要是III, IV 族元素), $X$ 代表碳元素或氮 元素, 目前已发现的 MAX 材料已超过 70 种 ${ }^{[7-8]}$, 而 MXenes 材料是将 MAX 相中 A 位元素抽出所形成 的二维材料, 其通式为 $\mathrm{M}_{n+1} \mathrm{X}_{n}(n=1,2 \text { 或 } 3)^{[9]}$ 。 MXenes 材料通常是在水溶液中选择性刻蚀获得的, 这使得 MXenes 材料表面往往含有大量 $-\mathrm{OH} 、-\mathrm{F}$ 等 官能团, 所以 MXenes 材料又记为 $\mathrm{M}_{n+1} \mathrm{X}_{n} \mathrm{~T}_{x}$ ( $\mathrm{T}$ 表示 $-\mathrm{OH} 、-\mathrm{F}$ 等官能团, $x$ 表示官能团数目 $)^{[10-11]}$ 。研究 表明大部分 MXenes 材料具有金属导电性，良好的 力学和减磨性能 ${ }^{[12-14]}$, 而 $\mathrm{Ti}_{3} \mathrm{C}_{2} \mathrm{~T}_{x}$ 作为研究最多的 MXenes 材料，已被应用于多种树脂基复合材料。 Shahzad 等 ${ }^{[15]}$ 制备的 $\mathrm{Ti}_{3} \mathrm{C}_{2} \mathrm{~T}_{x}$-SA(海藻酸钠)复合材 料表现出良好的电磁屏蔽效应; Cao 等 ${ }^{[16]}$ 研究发现 在聚偏二乙烯中添加 $5 \mathrm{wt} \%$ 的 $\mathrm{Ti}_{3} \mathrm{C}_{2} \mathrm{~T}_{x}$ 可以使其复合 材料的储能模量提高 $64 \%$; Zhang 等 ${ }^{[17]}$ 研究发现在 高分子复合材料中添加 $\mathrm{Ti}_{3} \mathrm{C}_{2} \mathrm{~T}_{x}$ 可以改善复合材料 的导热性、力学性能及耐磨性能。然而, 在铜基复 合材料领域还鲜有以 $\mathrm{Ti}_{3} \mathrm{C}_{2} \mathrm{~T}_{x}$ 为增强相的报道。

本工作通过分子级混合工艺及 SPS 烧结技术制 备 $\mathrm{Cu} / \mathrm{Ti}_{3} \mathrm{C}_{2} \mathrm{~T}_{x}$ 复合材料, 初步探索了 $\mathrm{Ti}_{3} \mathrm{C}_{2} \mathrm{~T}_{x}$ 含量对 铜基复合材料的力学、电学及摩擦磨损性能的影响。

\section{1 实验方法}

\section{1 实验原料}

$\mathrm{Ti}$ 粉 $(99.9 \%, 48 \mu \mathrm{m})$ 及 $\mathrm{Al}$ 粉 $(99.9 \%, 48 \mu \mathrm{m})$ 采 购自北京有色金属研究总院; 鳞片石墨 $(99.9 \%, 300$ 目)购自青岛天盛达石墨有限公司; 三水合硝酸铜 $\left(\mathrm{CuNO}_{3} \cdot 3 \mathrm{H}_{2} \mathrm{O}, \mathrm{AR}\right)$ 、葡萄糖 $\left(\mathrm{C}_{6} \mathrm{H}_{12} \mathrm{O}_{6}, \mathrm{AR}\right)$ 及氢氧化 钠 $(\mathrm{NaOH}, \mathrm{AR})$ 均购自上海阿拉丁生化科技有限公司; 氯化钠 $(\mathrm{NaCl}, \mathrm{AR})$ 、氯化钾 $(\mathrm{KCl}, \mathrm{AR})$ 及氢氟酸 $(\mathrm{HF}$, $\geqslant 40 \mathrm{wt} \%, \mathrm{AR})$ 购自国药集团化学试剂有限公司。

\section{2 复合材料制备}

\subsection{1 $\mathrm{Ti}_{3} \mathrm{C}_{2} \mathrm{~T}_{x}$ 材料制备}

采用熔盐法直接合成了 $\mathrm{Ti}_{3} \mathrm{AlC}_{2}$ 粉体, 具体工 艺过程如下: 将 $\mathrm{Ti} 、 \mathrm{C} 、 \mathrm{Al}$ 的元素粉按摩尔比为 $3.0: 2.0: 1.2$ 放入 $\mathrm{NaCl}-\mathrm{KCl}(1: 1, \mathrm{~mol} / \mathrm{mol})$ 熔盐体系 中, 并混合均匀; 将含熔盐的混合粉体置于 $1100^{\circ} \mathrm{C}$ 下 $\mathrm{Ar}$ 气氛中充分反应, 反应完毕后取出反应产物, 用去离子水多次洗涤后烘干, 即得 $\mathrm{Ti}_{3} \mathrm{AlC}_{2}$ 粉体。

取一定量的 $\mathrm{Ti}_{3} \mathrm{AlC}_{2}$ 粉体, 放在 $40 \mathrm{vol} \%$ 的 $\mathrm{HF}$ 酸溶液中, 室温下震荡浸泡两周, 经多次洗涤后真 空烘干, 即得 $\mathrm{Ti}_{3} \mathrm{C}_{2} \mathrm{~T}_{x}$ 粉体材料。

\subsection{2 $\mathrm{Cu} / \mathrm{Ti}_{3} \mathrm{C}_{2} \mathrm{~T}_{x}$ 复合材料制备}

采用分子级混合工艺制备 $\mathrm{Cu} / \mathrm{Ti}_{3} \mathrm{C}_{2} \mathrm{~T}_{x}$ 复合材料
粉体, 具体工艺过程如下: (1)对含有 $\mathrm{Ti}_{3} \mathrm{C}_{2} \mathrm{~T}_{x}$ 的铜盐 的乙醇溶液磁力搅拌 $3 \mathrm{~h}$; (2)加入葡萄糖溶液继续 搅拌 $2 \mathrm{~h}$; (3) 在超声作用下缓慢加入氢氧化钠溶液; (4)在 $60^{\circ} \mathrm{C}$ 下保温反应 $4 \mathrm{~h}$, 经多次洗涤后干燥、还 原即得 $\mathrm{Cu} / \mathrm{Ti}_{3} \mathrm{C}_{2} \mathrm{~T}_{x}$ 复合材料粉体; (5)采用 SPS 烧结 块体 $\mathrm{Cu} / \mathrm{Ti}_{3} \mathrm{C}_{2} \mathrm{~T}_{x}$ 复合材料, 烧结温度为 $800^{\circ} \mathrm{C}$, 升温 速率为 $50^{\circ} \mathrm{C} / \mathrm{min}$, 保温时间为 $5 \mathrm{~min}$, 保温压力为 $35 \mathrm{MPa}$, 降温时压力升至 $40 \mathrm{MPa}$ 。

\section{3 测试方法}

采用 D8 Advance 型 X 射线分析仪及 FEG 250 型扫描电子显微镜对 $\mathrm{Ti}_{3} \mathrm{C}_{2} \mathrm{~T}_{x} 、 \mathrm{Cu} / \mathrm{Ti}_{3} \mathrm{C}_{2} \mathrm{~T}_{x}$ 的物相及 微观形貌进行分析。利用 InVia-reflex 型拉曼光谱仪 及 FD-120 涡流电导仪分别对 $\mathrm{Cu} / \mathrm{Ti}_{3} \mathrm{C}_{2} \mathrm{~T}_{x}$ 复合材料 进行拉曼分析及导电性测试。 $\mathrm{Cu} / \mathrm{Ti}_{3} \mathrm{C}_{2} \mathrm{~T}_{x}$ 复合材料 的拉伸强度在 XQ-1 型纤维强度仪上进行测试。 $\mathrm{Cu} / \mathrm{Ti}_{3} \mathrm{C}_{2} \mathrm{~T}_{x}$ 复合材料摩擦性能在 UMT-3 高真空摩擦 磨损试验机上进行测试, 实验条件为: 将 $3 \mathrm{~mm}$ 大 小的 $\mathrm{Al}_{2} \mathrm{O}_{3}$ 球作为对立面球, 摩擦运动长度为 $5 \mathrm{~mm}$, 滑动速度为 $0.05 \mathrm{~m} / \mathrm{s}$, 施加负载为 $5 \mathrm{~N}$, 持续时间为 $30 \mathrm{~min}$, 测试环境湿度为 $60 \%$, 温度为 $16^{\circ} \mathrm{C}$ 。借助 于台阶仪计算摩擦实验磨损率, 利用台阶仪对磨痕 横截面进行扫描, 得到磨痕截面轮廓图。通过对磨 痕轮廓积分可得磨痕截面面积 $A$, 将截面面积 $A$ 带 入公式(1)即可得到样品的磨损率 $I$ 。

$$
I=(A \cdot l) /(N \cdot L)
$$

其中: $l$ 是磨痕长度, $L$ 是摩擦距离, $N$ 是加载载荷。

\section{2 结果与讨论}

\section{1 物相及微观形貌}

图 1 为 $\mathrm{Ti}_{3} \mathrm{C}_{2} \mathrm{~T}_{x}$ 的 XRD 图谱与 SEM 照片。由 图 1(a)可知, 经 $\mathrm{HF}$ 刻蚀后, $\mathrm{Ti}_{3} \mathrm{AlC}_{2}$ 相的低位峰向左 偏移, 说明随着 $\mathrm{Al}$ 原子被抽出, $\mathrm{Ti}_{3} \mathrm{AlC}_{2}$ 相腐蚀产物 的层间距变大。在 $2 \theta=8^{\circ}$ 左右出现的(0002)衍射峰对 应 $\mathrm{Ti}_{3} \mathrm{C}_{2} \mathrm{~T}_{x}$ 相的特征衍射峰。由图 1(b)可观察到大量 层状堆叠的 $\mathrm{Ti}_{3} \mathrm{C}_{2} \mathrm{~T}_{x}$ 片。

图 2 为 $\mathrm{Cu} / \mathrm{Ti}_{3} \mathrm{C}_{2} \mathrm{~T}_{x}$ 复合材料块体的 XRD 图谱, 由图可以看出, 当 $\mathrm{Ti}_{3} \mathrm{C}_{2} \mathrm{~T}_{x}$ 含量为 $5 \mathrm{vol} \%$ 时, 只有 $\mathrm{Cu}$ 的 XRD 衍射峰; 随着 $\mathrm{Ti}_{3} \mathrm{C}_{2} \mathrm{~T}_{x}$ 含量增加, 逐渐出现 $\mathrm{TiO}_{2}$ 相衍射峰; $\mathrm{Ti}_{3} \mathrm{C}_{2} \mathrm{~T}_{x}$ 含量越高, $\mathrm{TiO}_{2}$ 相的衍射峰 强度越强。这是由 $\mathrm{Ti}_{3} \mathrm{C}_{2} \mathrm{~T}_{x}$ 在粉体制备的碱性环境中 氧化形成的。而所有样品中均为未检测到 $\mathrm{Ti}_{3} \mathrm{C}_{2} \mathrm{~T}_{x}$ 相的衍射峰, 这是由于 $\mathrm{Cu}$ 基底的 XRD 衍射峰太强, 将其掩盖造成的。

图 3 为 $\mathrm{Cu} / \mathrm{Ti}_{3} \mathrm{C}_{2} \mathrm{~T}_{x}$ 复合材料的断面微观形貌。由 图 3(a)、(b)和(c)可知, $\mathrm{Ti}_{3} \mathrm{C}_{2} \mathrm{~T}_{x}$ 在铜基体中均匀分布, 

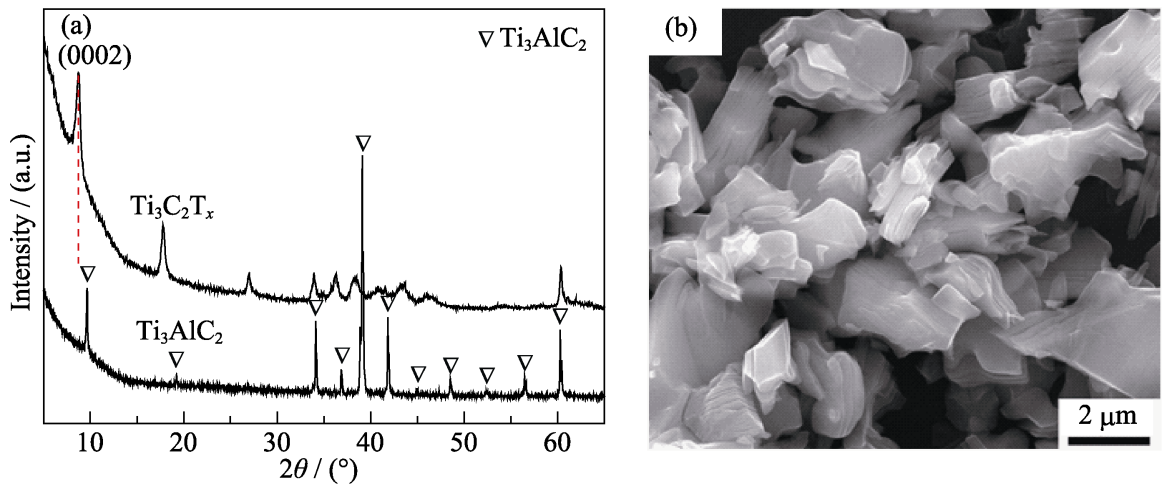

图 $1 \mathrm{Ti}_{3} \mathrm{C}_{2} \mathrm{~T}_{x}$ 的 XRD 图谱(a)与 $\mathrm{SEM}$ 照片(b)

Fig. 1 XRD pattern (a) and SEM image (b) of $\mathrm{Ti}_{3} \mathrm{C}_{2} \mathrm{~T}_{x}$

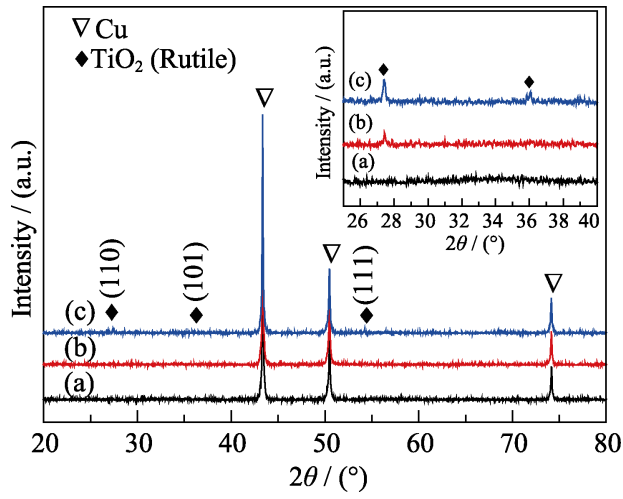

图 2 块体 $\mathrm{Cu} / \mathrm{Ti}_{3} \mathrm{C}_{2} \mathrm{~T}_{x}$ 复合材料的 XRD 图谱

Fig. 2 XRD patterns of $\mathrm{Cu} / \mathrm{Ti}_{3} \mathrm{C}_{2} \mathrm{~T}_{x}$ bulk composites

(a) $\mathrm{Cu} / 5 \mathrm{vol} \% \mathrm{Ti}_{3} \mathrm{C}_{2} \mathrm{~T}_{x}$; (b) $\mathrm{Cu} / 10 \mathrm{vol} \% \mathrm{Ti}_{3} \mathrm{C}_{2} \mathrm{~T}_{x}$; (c) $\mathrm{Cu} / 20 \mathrm{vol} \% \mathrm{Ti}_{3} \mathrm{C}_{2} \mathrm{~T}_{x}$

随着 $\mathrm{Ti}_{3} \mathrm{C}_{2} \mathrm{~T}_{x}$ 含量增加, 断面形貌中韧窝数量逐渐 较少, 说明 $\mathrm{Cu} / \mathrm{Ti}_{3} \mathrm{C}_{2} \mathrm{~T}_{x}$ 复合材料的塑性逐渐变差。
由图 3(d)、(e)和(f)可知, 随着 $\mathrm{Ti}_{3} \mathrm{C}_{2} \mathrm{~T}_{x}$ 含量增加, 断 面形貌中气孔增多, 说明 $\mathrm{Cu} / \mathrm{Ti}_{3} \mathrm{C}_{2} \mathrm{~T}_{x}$ 复合材料的致 密性逐渐降低。由图 3(f)中可观察到大量白色颗粒状 物质, 结合 XRD 分析其为金红石 $\mathrm{TiO}_{2}$ 。另外, 图 3 中 $\mathrm{Ti}_{3} \mathrm{C}_{2} \mathrm{~T}_{x}$ 形貌与图 1 中 $\mathrm{Ti}_{3} \mathrm{C}_{2} \mathrm{~T}_{x}$ 表现出明显不同。 图 1 中原始 $\mathrm{Ti}_{3} \mathrm{C}_{2} \mathrm{~T}_{x}$ 为 “书页” 状层状分离形貌, 且 $\mathrm{Ti}_{3} \mathrm{C}_{2} \mathrm{~T}_{x}$ 片表面光滑; 而图 3 中 $\mathrm{Ti}_{3} \mathrm{C}_{2} \mathrm{~T}_{x}$ 中层状分离 结构消失, $\mathrm{Ti}_{3} \mathrm{C}_{2} \mathrm{~T}_{x}$ 表面粗糙。这种结构形貌的转化 与复合材料制备过程中 $\mathrm{Ti}_{3} \mathrm{C}_{2} \mathrm{~T}_{x}$ 材料表面官能团的 变化及烧结过程中与铜的相互作用有关。

\section{2 性能分析}

图 4 为 $\mathrm{Cu} / \mathrm{Ti}_{3} \mathrm{C}_{2} \mathrm{~T}_{x}$ 复合材料的电导率及相对密 度随 $\mathrm{Ti}_{3} \mathrm{C}_{2} \mathrm{~T}_{x}$ 含量的变化曲线。由图 4 可知, 随着 $\mathrm{Ti}_{3} \mathrm{C}_{2} \mathrm{~T}_{x}$ 含量增加, $\mathrm{Cu} / \mathrm{Ti}_{3} \mathrm{C}_{2} \mathrm{~T}_{x}$ 复合材料的相对密度及
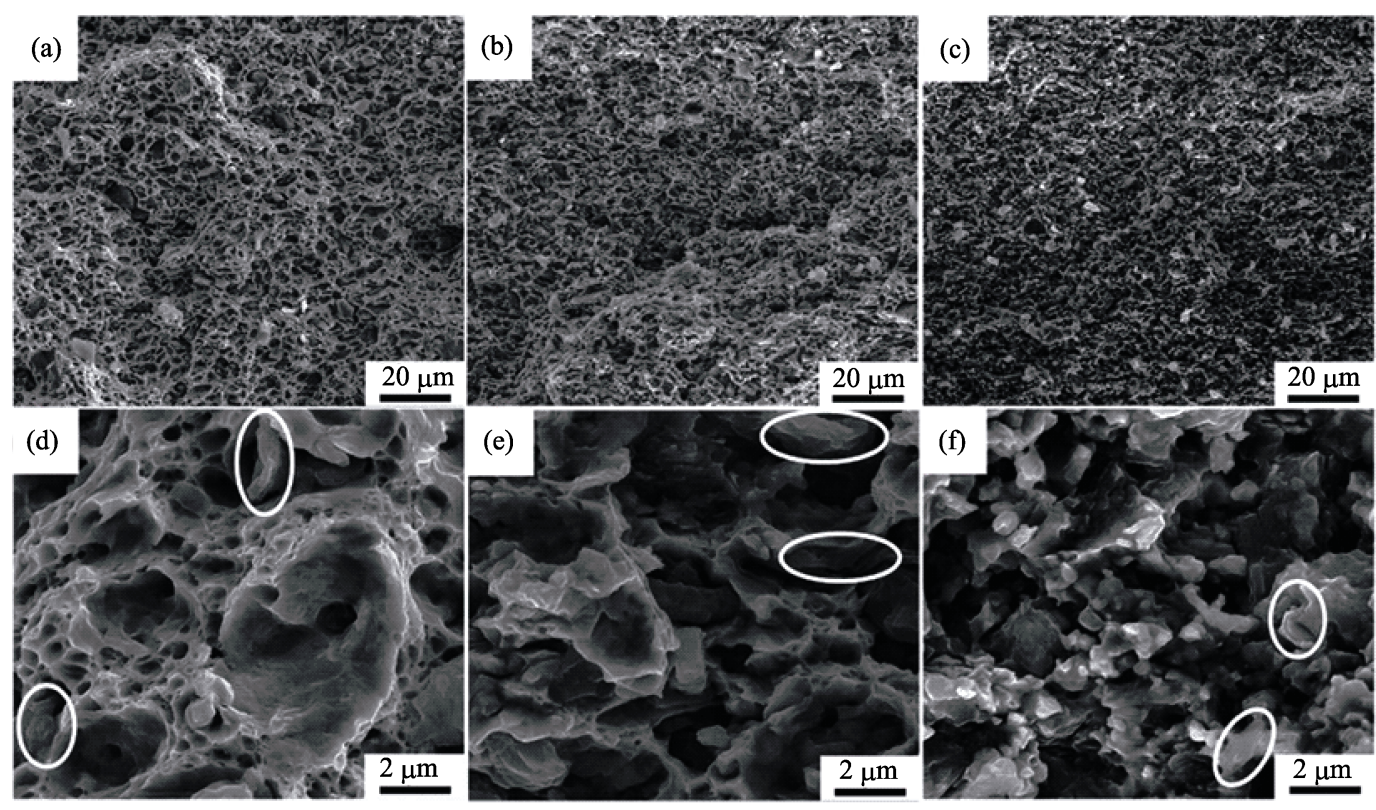

图 $3 \mathrm{Cu} / \mathrm{Ti}_{3} \mathrm{C}_{2} \mathrm{~T}_{x}$ 复合材料断面的 SEM 照片

Fig. 3 SEM images of $\mathrm{Cu} / \mathrm{Ti}_{3} \mathrm{C}_{2} \mathrm{~T}_{x}$ composites (a, d) Cu/5vol\% $\mathrm{Ti}_{3} \mathrm{C}_{2} \mathrm{~T}_{x}$; (b, e) Cu/10vol\% $\mathrm{Ti}_{3} \mathrm{C}_{2} \mathrm{~T}_{x}$; (c, f) Cu/20vol\% $\mathrm{Ti}_{3} \mathrm{C}_{2} \mathrm{~T}_{x}$ 


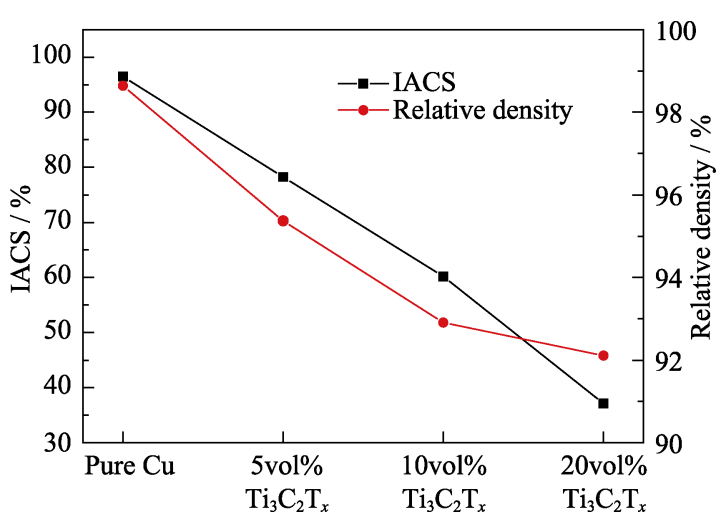

图 $4 \mathrm{Cu} / \mathrm{Ti}_{3} \mathrm{C}_{2} \mathrm{~T}_{x}$ 复合材料的相对密度及电导率随 $\mathrm{Ti}_{3} \mathrm{C}_{2} \mathrm{~T}_{x}$ 含量的变化曲线

Fig. 4 Electrical conductivity and relative density of $\mathrm{Cu} / \mathrm{Ti}_{3} \mathrm{C}_{2} \mathrm{~T}_{x}$ composites as a function of $\mathrm{Ti}_{3} \mathrm{C}_{2} \mathrm{~T}_{x}$ content

电导率逐渐降低。 $\mathrm{Ti}_{3} \mathrm{C}_{2} \mathrm{~T}_{x}$ 含量为 $5 \mathrm{vol} \%$ 时, $\mathrm{Cu} / \mathrm{Ti}_{3} \mathrm{C}_{2} \mathrm{~T}_{x}$ 复合材料的 IACS(国际退火铜标准(缩写 IACS)规定 的电阻率(体积或质量)与相同单位的试样电阻率之 比值)电导率有 $80 \%$; 当 $\mathrm{Ti}_{3} \mathrm{C}_{2} \mathrm{~T}_{x}$ 含量增加为 $20 \mathrm{vol} \%$ 时, 复合材料的 IACS 电导率下降为 37\%。增强相 的导电性、含量及复合材料相对密度直接影响着金 属基复合材料的导电性 ${ }^{[18-19]}$ 。随着 $\mathrm{Ti}_{3} \mathrm{C}_{2}$ 含量增加, 复合材料致密性变差, 存在的大量气孔降低了复合 材料的导电性; 相对铜基体 $\mathrm{Ti}_{3} \mathrm{C}_{2} \mathrm{~T}_{x}$ 本身的导电性 差，而 $\mathrm{Ti}_{3} \mathrm{C}_{2} \mathrm{~T}_{x}$ 含量越高时，铜基体结构破坏越严重， 阻断了基体结构中的电子传输。另外, 随着 $\mathrm{Ti}_{3} \mathrm{C}_{2} \mathrm{~T}_{x}$ 含量增加, 复合材料中 $\mathrm{TiO}_{2}$ 含量增加, 生成的 $\mathrm{TiO}_{2}$ 进一步恶化了复合材料的导电性。

图 5 为不同 $\mathrm{Ti}_{3} \mathrm{C}_{2} \mathrm{~T}_{x}$ 含量的 $\mathrm{Cu} / \mathrm{Ti}_{3} \mathrm{C}_{2} \mathrm{~T}_{x}$ 复合材料 的应力-应变曲线图。由图 5 可知, 纯铜呈典型的塑 性断裂，其断裂延伸率超过 30\%。所有 $\mathrm{Cu} / \mathrm{Ti}_{3} \mathrm{C}_{2} \mathrm{~T}_{x}$ 复合材料样品均未表现出屈服现象, 呈明显脆性断 裂。随着 $\mathrm{Ti}_{3} \mathrm{C}_{2} \mathrm{~T}_{x}$ 含量增加, $\mathrm{Cu} / \mathrm{Ti}_{3} \mathrm{C}_{2} \mathrm{~T}_{x}$ 复合材料的 延伸率逐渐降低, 而拉伸强度则呈先升高后降低的 变化趋势。当 $\mathrm{Ti}_{3} \mathrm{C}_{2} \mathrm{~T}_{x}$ 含量为 $5 \mathrm{vol} \%$ 时, $\mathrm{Cu} / \mathrm{Ti}_{3} \mathrm{C}_{2} \mathrm{~T}_{x}$ 复合材料的最大拉伸应力为 $390 \mathrm{MPa}$, 相比纯铜提 高了 $44 \%$; 而 $\mathrm{Ti}_{3} \mathrm{C}_{2} \mathrm{~T}_{x}$ 含量为 $20 \mathrm{vol} \%$ 时, $\mathrm{Cu} / \mathrm{Ti}_{3} \mathrm{C}_{2} \mathrm{~T}_{x}$ 复合材料的最大拉伸应力不足 $175 \mathrm{MPa}$, 低于纯铜 的最大拉伸应力, 此时复合材料的力学性能已被严 重恶化。结合图 3(d)分析可知, 在拉伸过程中 $\mathrm{Ti}_{3} \mathrm{C}_{2} \mathrm{~T}_{x}$ 有被拔出迹象, 故 $\mathrm{Ti}_{3} \mathrm{C}_{2} \mathrm{~T}_{x}$ 可以起到一定的增强作 用。当 $\mathrm{Ti}_{3} \mathrm{C}_{2} \mathrm{~T}_{x}$ 含量增加时, $\mathrm{Cu} / \mathrm{Ti}_{3} \mathrm{C}_{2} \mathrm{~T}_{x}$ 复合材料的 基体结构被破坏, 导致铜基体无法有效地承载载荷, 从而造成复合材料性能恶化。另外, 当 $\mathrm{Ti}_{3} \mathrm{C}_{2} \mathrm{~T}_{x}$ 含量 增加时, 复合材料中气孔及杂相 $\mathrm{TiO}_{2}$ 含量增加, 进 一步造成了 $\mathrm{Cu} / \mathrm{Ti}_{3} \mathrm{C}_{2} \mathrm{~T}_{x}$ 复合材料的力学性能恶化。
图 6 为 $\mathrm{Cu} / \mathrm{Ti}_{3} \mathrm{C}_{2} \mathrm{~T}_{x}$ 复合材料的摩擦系数随滑移 时间的变化, 由图 6 可知, $\mathrm{Cu} / \mathrm{Ti}_{3} \mathrm{C}_{2} \mathrm{~T}_{x}$ 复合材料的摩 擦系数呈先减小后增大的变化趋势。当 $\mathrm{Ti}_{3} \mathrm{C}_{2} \mathrm{~T}_{x}$ 含量 为 $5 \mathrm{vol} \%$ 时, $\mathrm{Cu} / \mathrm{Ti}_{3} \mathrm{C}_{2} \mathrm{~T}_{x}$ 复合材料的摩擦系数最小约 为 0.26 ; 当 $\mathrm{Ti}_{3} \mathrm{C}_{2} \mathrm{~T}_{x}$ 含量为 $20 \mathrm{vol} \%$ 时, $\mathrm{Cu} / \mathrm{Ti}_{3} \mathrm{C}_{2} \mathrm{~T}_{x}$ 复 合材料的摩擦系数略高于纯铜样品的摩擦系数。由 表 1 可知, $\mathrm{Cu} / \mathrm{Ti}_{3} \mathrm{C}_{2} \mathrm{~T}_{x}$ 复合材料的磨损率随着 $\mathrm{Ti}_{3} \mathrm{C}_{2} \mathrm{~T}_{x}$ 含量的增加先减小后增大。当 $\mathrm{Ti}_{3} \mathrm{C}_{2} \mathrm{~T}_{x}$ 含量 为 $10 \mathrm{vol} \%$ 时, $\mathrm{Cu} / \mathrm{Ti}_{3} \mathrm{C}_{2} \mathrm{~T}_{x}$ 复合材料的磨损率最小, 为 $2.55 \times 10^{-7} \mathrm{~mm}^{3} /(\mathrm{N} \cdot \mathrm{m})$, 比纯铜样品的磨损率小一 个数量级。以上结果表明: $\mathrm{Ti}_{3} \mathrm{C}_{2} \mathrm{~T}_{x}$ 含量为 $10 \mathrm{vol} \%$ 时, $\mathrm{Cu} / \mathrm{Ti}_{3} \mathrm{C}_{2} \mathrm{~T}_{x}$ 复合材料表现出最好的耐磨性, 即在铜 基复合材料中添加适量 $\mathrm{Ti}_{3} \mathrm{C}_{2} \mathrm{~T}_{x}$ 可以提高复合材料 的耐磨擦磨损性能。

图 7 为 $\mathrm{Cu} / \mathrm{Ti}_{3} \mathrm{C}_{2} \mathrm{~T}_{x}$ 复合材料磨痕微观形貌, 样 品表面可以观察到由犁削造成的划痕，这是由对磨 材料 $\mathrm{Al}_{2} \mathrm{O}_{3}$ 的表面凸起在摩擦运动中割据复合材料 表面造成的。图 7(a)中纯铜样品表面有大量微裂纹, 表层材料呈块状脱落, 这是因为摩擦运动是反复加

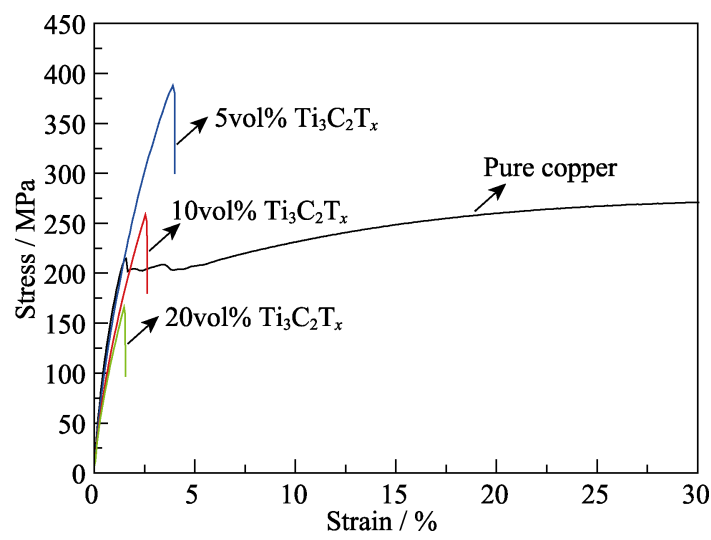

图 $5 \mathrm{Cu} / \mathrm{Ti}_{3} \mathrm{C}_{2} \mathrm{~T}_{x}$ 复合材料的应力一应变曲线图

Fig. 5 Stress-strain curves of $\mathrm{Cu} / \mathrm{Ti}_{3} \mathrm{C}_{2} \mathrm{~T}_{x}$ composites

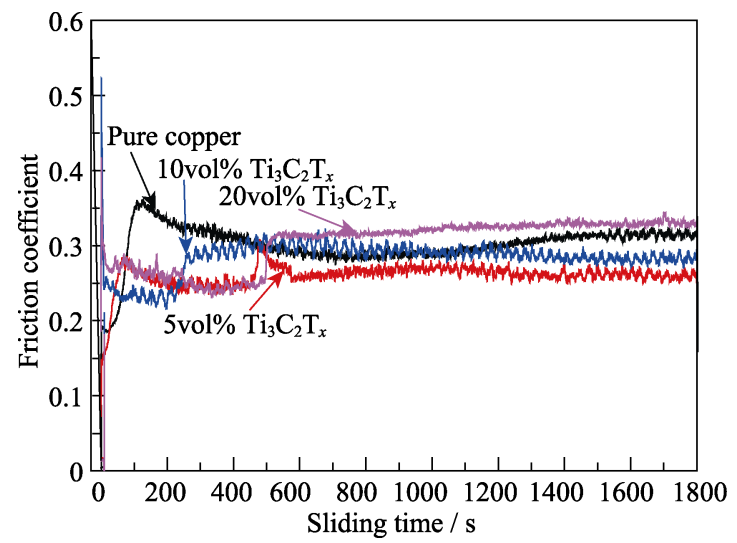

图 $6 \mathrm{Cu} / \mathrm{Ti}_{3} \mathrm{C}_{2} \mathrm{~T}_{x}$ 复合材料的摩擦系数随滑移时间的变化 Fig. 6 Change of friction coefficients for $\mathrm{Cu} / \mathrm{Ti}_{3} \mathrm{C}_{2} \mathrm{~T}_{x}$ composites with sliding time 
表 $1 \mathrm{Cu} / \mathbf{T i}_{3} \mathbf{C}_{2} \mathbf{T}_{x}$ 复合材料的磨损率

Table 1 Width of wear tracks and wear rates of $\mathrm{Cu} / \mathrm{Ti}_{3} \mathrm{C}_{2} \mathrm{~T}_{X}$ composites

\begin{tabular}{cc}
\hline Sample ID & Wear rate $/\left(\mathrm{mm}^{3} \cdot \mathrm{N}^{-1} \cdot \mathrm{m}^{-1}\right)$ \\
\hline Pure copper & $3.79 \times 10^{-6}$ \\
5 vol\% $\mathrm{Ti}_{3} \mathrm{C}_{2} \mathrm{~T}_{x}$ & $2.25 \times 10^{-6}$ \\
10vol\% $\mathrm{Ti}_{3} \mathrm{C}_{2} \mathrm{~T}_{x}$ & $2.55 \times 10^{-7}$ \\
$20 \mathrm{vol} \% \mathrm{Ti}_{3} \mathrm{C}_{2} \mathrm{~T}_{x}$ & $4.98 \times 10^{-6}$ \\
\hline
\end{tabular}
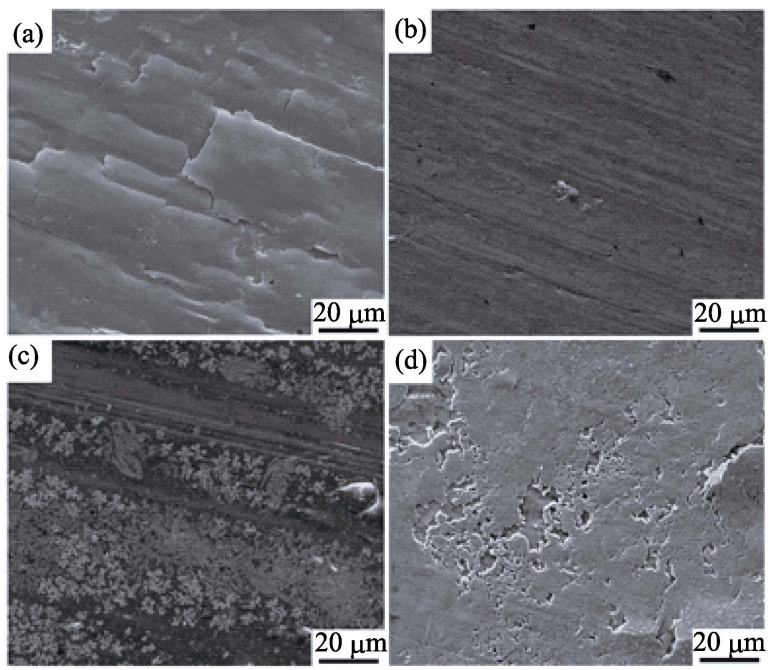

图 $7 \mathrm{Cu} / \mathrm{Ti}_{3} \mathrm{C}_{2} \mathrm{~T}_{x}$ 复合材料的磨痕微观形貌

Fig. 7 SEM images of the wear tracks of $\mathrm{Cu} / \mathrm{Ti}_{3} \mathrm{C}_{2} \mathrm{~T}_{x}$ composites

(a) $\mathrm{Cu}$; (b) $\mathrm{Cu} / 5 \mathrm{vol} \% \mathrm{Ti}_{3} \mathrm{C}_{2} \mathrm{~T}_{x}$; (c) $\mathrm{Cu} / 10 \mathrm{vol} \% \mathrm{Ti}_{3} \mathrm{C}_{2} \mathrm{~T}_{x}$; (d) $\mathrm{Cu} / 20 \mathrm{vol} \%$ $\mathrm{Ti}_{3} \mathrm{C}_{2} \mathrm{~T}_{x}$

载-卸载的循环过程, 当循环应力超过纯铜表层材 料的极限疲劳强度时, 在表层材料应力集中区域产生 微裂纹并扩展，最终导致表层材料剥离脱落。图 7(b) 与(c)中样品表面光滑, 仅有少量因粘着磨损造成的 脱落, 并且图 7(c)中复合材料表面可观察到大量 $\mathrm{Ti}_{3} \mathrm{C}_{2} \mathrm{~T}_{x}$, 在施加压力作用下层状 $\mathrm{Ti}_{3} \mathrm{C}_{2} \mathrm{~T}_{x}$ 会随着滑 移运动发生变形, 由于非化学计量比 $\mathrm{TiC}$ 与铜有较 好的润湿性 ${ }^{[20]}$, 剥离的 $\mathrm{Ti}_{3} \mathrm{C}_{2} \mathrm{~T}_{x}$ 在摩擦表面堆积形 成润滑膜，从而起到一定的减磨润滑作用，所以 $\mathrm{Cu} / 10 \mathrm{vol} \% \mathrm{Ti}_{3} \mathrm{C}_{2} \mathrm{~T}_{x}$ 复合材料表现出最低的磨损率。

图 7(d)中样品表面有大量微裂纹和因表层材料脱落 而留下的空洞, 说明复合材料的磨损程度加剧。由 $\mathrm{Cu} / 20 \mathrm{vol} \% \mathrm{Ti}_{3} \mathrm{C}_{2} \mathrm{~T}_{x}$ 复合材料磨痕处的拉曼分析结果 (见图 8)可知, 在摩擦样品表面可以检测到 Ti-C 振 动峰 ${ }^{[9]}$, 说明摩擦样品表层中仍然存在 $\mathrm{Ti}_{3} \mathrm{C}_{2} \mathrm{~T}_{x}$ 。另 外在样品表层可检测到较强的 $\mathrm{A}(\mathrm{Ti}-\mathrm{O})$ 和 $\mathrm{R}(\mathrm{Ti}-\mathrm{O})$ 振动峰 ${ }^{[21-22]}$, 说明摩擦产物中存在大量 $\mathrm{TiO}_{2}$ 。由于 摩擦前样品的 XRD 图谱只检测到金红石 $\mathrm{TiO}_{2}$, 所 以锐钛矿 $\mathrm{TiO}_{2}$ 应该是在摩擦中产生的。摩擦中生成 的大量 $\mathrm{TiO}_{2}$ 加剧了 $\mathrm{Cu} / \mathrm{Ti}_{3} \mathrm{C}_{2} \mathrm{~T}_{x}$ 的磨损过程, 所以 $\mathrm{Cu} / 20 \mathrm{vol} \% \mathrm{Ti}_{3} \mathrm{C}_{2} \mathrm{~T}_{x}$ 复合材料表现出较高的磨损率。

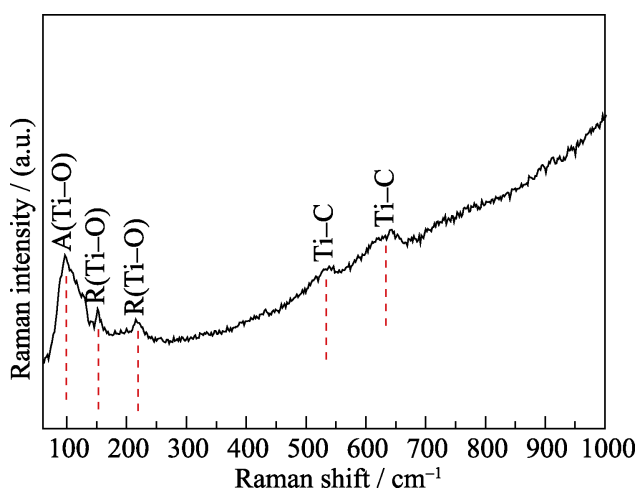

图 $8 \mathrm{Cu} / 20 \mathrm{vol} \% \mathrm{Ti}_{3} \mathrm{C}_{2} \mathrm{~T}_{x}$ 复合材料样品磨痕处的拉曼图谱 Fig. 8 Raman spectra of $\mathrm{Cu} / 20 \mathrm{vol} \% \mathrm{Ti}_{3} \mathrm{C}_{2} \mathrm{~T}_{x}$ composites at the wear track

\section{3 结论}

通过对不同 $\mathrm{Ti}_{3} \mathrm{C}_{2} \mathrm{~T}_{x}$ 含量的 $\mathrm{Cu} / \mathrm{Ti}_{3} \mathrm{C}_{2} \mathrm{~T}_{x}$ 复合材料 的微观形貌、导电性、拉伸性能及摩擦磨损性能进 行分析，得到以下结论：

1) 随着 $\mathrm{Ti}_{3} \mathrm{C}_{2} \mathrm{~T}_{x}$ 含量增加, $\mathrm{Cu} / \mathrm{Ti}_{3} \mathrm{C}_{2} \mathrm{~T}_{x}$ 复合材料 的导电性下降。这是由 $\mathrm{Ti}_{3} \mathrm{C}_{2} \mathrm{~T}_{x}$ 本身的导电性差和复 合材料的相对密度降低造成的。

2) 随着 $\mathrm{Ti}_{3} \mathrm{C}_{2} \mathrm{~T}_{x}$ 含量增加, $\mathrm{Cu} / \mathrm{Ti}_{3} \mathrm{C}_{2} \mathrm{~T}_{x}$ 复合材料 的拉伸强度先增加后下降。这是由于 $\mathrm{Ti}_{3} \mathrm{C}_{2} \mathrm{~T}_{x}$ 与铜 有一定的润湿能力, $\mathrm{Ti}_{3} \mathrm{C}_{2} \mathrm{~T}_{x}$ 含量较少时可以强化复 合材料界面，在拉伸中承载一定力，从而提高复合 材料的拉伸强度，而当 $\mathrm{Ti}_{3} \mathrm{C}_{2} \mathrm{~T}_{x}$ 含量过高时，铜基的 结构破坏越严重, 恶化了复合材料的力学性能。

3) 随着 $\mathrm{Ti}_{3} \mathrm{C}_{2} \mathrm{~T}_{x}$ 含量增加, 复合材料的摩擦系数 及磨损率均呈现先减少后增加的变化趋势, 当 $\mathrm{Ti}_{3} \mathrm{C}_{2} \mathrm{~T}_{x}$ 含量为 $10 \mathrm{vol} \%$ 时, $\mathrm{Cu} / \mathrm{Ti}_{3} \mathrm{C}_{2} \mathrm{~T}_{x}$ 复合材料表现出最好 的耐磨损性。这是因为 $\mathrm{Ti}_{3} \mathrm{C}_{2} \mathrm{~T}_{x}$ 为片层结构, 在压 力作用下可以沿片层方向发生滑动从而起到润滑 的作用。

\section{参考文献:}

[1] CHEN YA-KUN, ZHANG XIANG, LIU EN-ZUO, et al. Fabrication of in-situ grown graphene reinforced $\mathrm{Cu}$ matrix composites. Scientific Reports, 2016, 6: 19363.

[2] WANG LI-DONG, CUI YE, YANG SHUAI, et al. Microstructure and properties of carbon nanosheet/copper composites processed by particle-assisted shear exfoliation. RSC Advances, 2015, 5(25): 19321-19328.

[3] KOVALCHENKO A M, FUSHCHICH O I, DANYLUK S. The tribological properties and mechanism of wear of Cu-based sintered powder materials containing molybdenum disulfide and molybdenum diselenite under unlubricated sliding against copper. Wear, 2012, 290-291: 106-123.

[4] PAWLAK Z, KALDONSKI T, PAI R, et al. A comparative study on the tribological behaviour of hexagonal boron nitride ( $h$ - $\mathrm{BN})$ as 
lubricating micro-particles-an additive in porous sliding bearings for a car clutch. Wear, 2009, 267(5/8): 1198-1202.

[5] CHEN FAN-YAN, YING JIA-MIN, WANG YI-FEI, et al. Effects of graphene content on the microstructure and properties of copper matrix composites. Carbon, 2016, 96: 836-842.

[6] HONG NG VINCENT-MING, HUANG HUI, ZHOU KUN, et al. Recent progress in layered transition metal carbides and/or nitrides (MXenes) and their composites: synthesis and applications. Journal of Materials Chemistry A, 2017, 5(7): 3039-3068.

[7] BARSOUM M W. MAX Phases: Properties of Machinable Ternary Carbides and Nitrides. John Wiley \& Sons, 2013: 1-5.

[8] LAPAUW T, LAMBRINOU K, CABIOC'H T, et al. Synthesis of the new MAX phase $\mathrm{Zr}_{2} \mathrm{AlC}$. Journal of the European Ceramic Society, 2016, 36(8): 1847-1853.

[9] NAGUIB MICHAEL, KURTOGLU MURAT, PRESSER VOLKER, et al. Two-dimensional nanocrystals produced by exfoliation of $\mathrm{Ti}_{3} \mathrm{AlC}_{2}$. Advanced Materials, 2011, 23(37): 4248-4253.

[10] ZHANG TIAN, PAN LI-MEI, TANG HUAN, et al. Synthesis of two-dimensional $\mathrm{Ti}_{3} \mathrm{C}_{2} \mathrm{~T}_{x}$ MXene using $\mathrm{HCl}+\mathrm{LiF}$ etchant: enhanced exfoliation and delamination. Journal of Alloys and Compounds, 2017, 695: 818-826.

[11] LIU FAN-FAN, ZHOU AI-GUO, CHEN JIN-FENG, et al. Preparation of $\mathrm{Ti}_{3} \mathrm{C}_{2}$ and $\mathrm{Ti}_{2} \mathrm{C}$ MXenes by fluoride salts etching and methane adsorptive properties. Applied Surface Science, 2017, 416: 781-789.

[12] NAGUIB MICHAEL, MOCHALIN VADYM N, BARSOUM MICHEL W, et al. 25th anniversary article: MXenes: a new family of two-dimensional materials. Advanced Materials, 2014, 26(7): 992-1005.

[13] HALIM JOSEPH, LUKATSKAYA MARIA R, COOK KEVIN M, et al. Transparent conductive two-dimensional titanium carbide epitaxial thin films. Chemistry of Materials, 2014, 26(7): 23742381.
[14] YANG JIN, CHEN BEI-BEI, SONG HAO-JIE, et al. Synthesis, characterization, and tribological properties of two-dimensional $\mathrm{Ti}_{3} \mathrm{C}_{2}$. Crystal Research and Technology, 2014, 49(11): 926-932.

[15] SHAHZAD FAISAL, ALHABEB MOHAMED, HATTER CHRISTINE B, et al. Electromagnetic interference shielding with 2D transition metal carbides (MXenes). Science, 2016, 353(6304): 1137-1140.

[16] CAO YONG, DENG QI-HUANG, LIU ZHI-DUO, et al. Enhanced thermal properties of poly(vinylidene fluoride) composites with ultrathin nanosheets of MXene. RSC Advances., 2017, 7(33): 2049420501.

[17] ZHANG HENG, WANG LI-BO, CHEN QIANG, et al. Preparation, mechanical and anti-friction performance of MXene/polymer composites. Materials \& Design, 2016, 92: 682-689.

[18] WEBER L, DORN J, MORTENSEN A. On the electrical conductivity of metal matrix composites containing high volume fractions of non-conducting inclusions. Acta Materialia, 2003, 51(11): 3199-3211.

[19] WEI BING-WEI W, QU DONG, HU CHUN-FENG, et al. Synthesis and physical properties of graphene nanosheets reinforced copper composites. Advanced Materials Research, 2013, 833: 310-314.

[20] ZARRINFAR N, KENNEDY A R, SHIPWAY P H. Reaction synthesis of $\mathrm{Cu}-\mathrm{TiC}_{x}$ master-alloys for the production of copper-based composites. Scripta Materialia, 2004, 50(7): 949-952.

[21] NAGUIB MICHAEL, MASHTALIR OLHA, LUKATSKAYA MARIA R, et al. One-step synthesis of nanocrystalline transition metal oxides on thin sheets of disordered graphitic carbon by oxidation of MXenes. Chemical Communications, 2014, 50(56): 7420-7423.

[22] GHASSEMIh H, HARLOW W, MASHTALIR O, et al. In situ environmental transmission electron microscopy study of oxidation of two-dimensional $\mathrm{Ti}_{3} \mathrm{C}_{2}$ and formation of carbon-supported $\mathrm{TiO}_{2}$. Journal of Materials Chemistry A, 2014, 2(35): 14339. 